\title{
Heavy Element Synthesis and Cosmology
}

\author{
Mounib El Eid \\ American University of Beirut, \\ Department of Physics \\ PO Box 11=0236, Riad El-Solh 1107 2020, Beirut, Lebanon \\ E-mail: meideaub. edu. Ib
}

\begin{abstract}
We present a brief summary of the abundances of heavy element as a function of metallicity. In particular, the abundance pattern of these elements at very low metallicity has received a great deal of interest, because they may indicate how the heavy-element nucleosynthesis has evolved during the history of the Galaxy. In addition, the understanding of the heavy element distribution in very metal-poor halo stars is a powerful tool to learn about the formation of the element during the early phase of the Galaxy and even during a pre-galactic epoch. A challenging problem is to investigate the role of the first stars in synthesizing the heavy elements. This will be subject of this contribution.
\end{abstract}

\section{Introduction}

The first stars formed after Big Bang were quite different from those formed later, simply because they were composed mainly of hydrogen and helium and traces of light elements up ${ }^{7} \mathrm{Li}$. The classification of the first stars is not easy. A classification scheme is by [1] as follows: Pop III.1 are stars which are formed under initial cosmological parameters, Pop III.2 are stars formed from photo-ionized gas by earlier star generation(s). The pop II stars may be divided into 3 classes: EMP (extremely-metal poor) stars with $10^{-4}<[\mathrm{Fe} / \mathrm{H}]<10^{-3}$, so that $[\mathrm{Fe} / \mathrm{H}]=0$ is solar, (see description of Fig. 1 for definition of $[\mathrm{Fe} / \mathrm{H}]), U M P$ (ultra-metal poor ) with $10^{-5}<[\mathrm{Fe} / \mathrm{H}]<10^{-4}$ and $H M P$ (hyper-metal poor) with $10^{-6}<[\mathrm{Fe} / \mathrm{H}]<10^{-5}$. It seems that such classification is related to the nature of the first galaxies, where a galaxy is a system of stars, gas and dark matter halo. Within this picture, pop III stars are assumed to be formed in isolation in mini halos, not in galaxies. Several arguments exist (see ref [1] for more details) that the first stars were massive and even very massive, mainly because of their formation in gas clouds devoid of heavy elements with higher internal pressure than in metal-rich clouds at the same temperature. In other words, cooling and effective fragmentation were inhibited in such metal-free clouds. If so, the first stars represents an extinct generation, because they ended in supernovae explosions, not necessarily as core collapse supernova, but eventually as pair-creation supernovae as we describe below in sect. 4 . Observing the chemical abundances in the oldest surviving stars is a way to learn about the nature of the first stars. But the chemical abundances are related to nucleosynthesis processes occurring in stars, and can be linked to stellar evolution. 
Heavy elements are formed in the universe by neutron capture processes beyond iron via the s-process nucleosynthesis mainly in Asymptotic Giant Branch Stars (AGB) and in pre-supernova evolution of massive stars, and by the r-process mainly in supernovae explosion of massive stars.

The metal-poor stars in the halo serve as "laboratory" for the study of the nucleosynthesis of neutron-capture elements. Their chemical compositions are linked to the types of synthesis processes that occurred during the early phase of the Galaxy.

The presence of heavy elements in the atmospheres of these stars indicates extinct generation of massive stars which have synthesized all heavy elements. Therefore, it is useful to see what the observations of heavy elements in the oldest stars look like. This is done in sect.2. In sect.3, a cosmic connection to the early stars is outlined. In sect. 4, some evolutionary scenarios are described in connection with the early nucleosynthesis of heavy elements. Concluding remarks are given in sect.5. .

\section{Heavy elements in oldest Stars}

As a first example the observation obtained for the r-process rich star CS22892-52 characterized by $[\mathrm{Ba} / \mathrm{Fe}]=+1.6\left([\mathrm{X} / \mathrm{Fe}]=\log (\mathrm{X} / \mathrm{Fe})_{\text {star }}-\log (\mathrm{X} / / \mathrm{Fe})_{\text {Sun }}\right)$ by [2]. Fig. 1 shows the results for this star which can be considered as EMP star with $[\mathrm{Fe} / \mathrm{H}]=-3.1$.

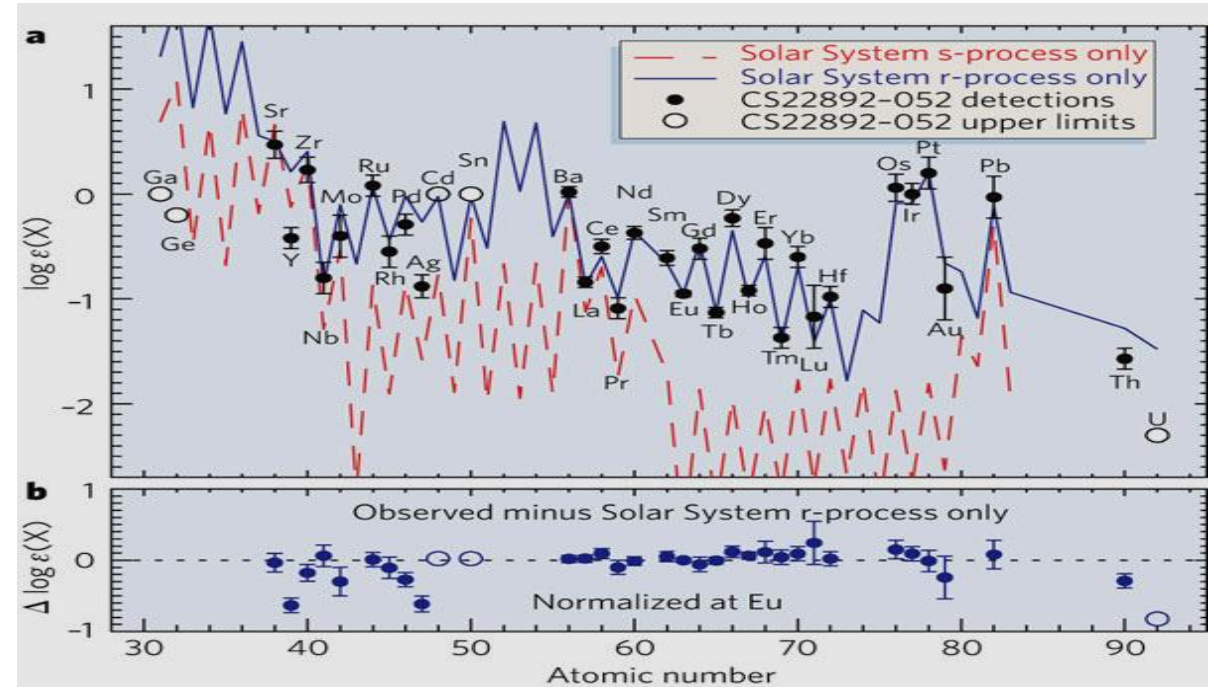

Fig.1: Abundances of neutron-capture elements obtained for the star CS22892-52 by [2]

Fig.1 shows remarkable features. The distribution of the neutron-capture elements having atomic number $\mathrm{Z} \geq 56$ matches the solar system r-process pattern, while that of the light r-process elements does not match the solar r-process pattern. For example, the element silver (Ag with $\mathrm{Z}=47$ ) considered as indicator for the r-process is deficient. It seems that the r-process could be divided, similarly to the s-process, into two components: a weak r-process (so far it is called LEPP (Light Element Primary Process), and Main r-process, or classical r-process. A similar case is the star CS31082001 according to the observation by [3].

Heavy elements have also been observed in ultra-metal poor stars having $[\mathrm{Fe} / \mathrm{H}]=-5.0$ like HE 0107-5420 and HE1327-2326. Both are rich in CNO, but very poor in neutron- 
capture elements. This is different from the previous cases (CS 22892-052 or CS31082001). The conclusion from these observations is that the first stars were massive and able to produce $\mathrm{CNO}$ elements, but not the heavier neutron-capture elements.

\subsection{Light neutron-capture Elements}

The lighter neutron-capture elements $(\mathrm{Z}<56)$ seem to fall below the solar system curve. Observation of four metal-poor r-enriched stars (see [4]) indicates that silver $(\mathrm{Ag}, \mathrm{Z}=47)$ is produced in proportion of the heavier elements in stars with $-2.2<[\mathrm{Fe} / \mathrm{H}]<$ -1.2. In the work by [5], it was suggested that not all the $\mathrm{Sr}$ to $\mathrm{Zr}$ solar abundances can be explained by the canonical r-process or s-process. An unknown process was postulated and termed "LEPP" (Light Element Primary Process) producing Sr, Y and Zr, but little $\mathrm{Eu}$ and $\mathrm{Ba}$. In the work by [6], the range of the LEPP was extended up to $\mathrm{Ba}$, or to the range of atomic number $\mathrm{Z}=-38-56$. The need for such a peculiar process is clearly in Fig.2

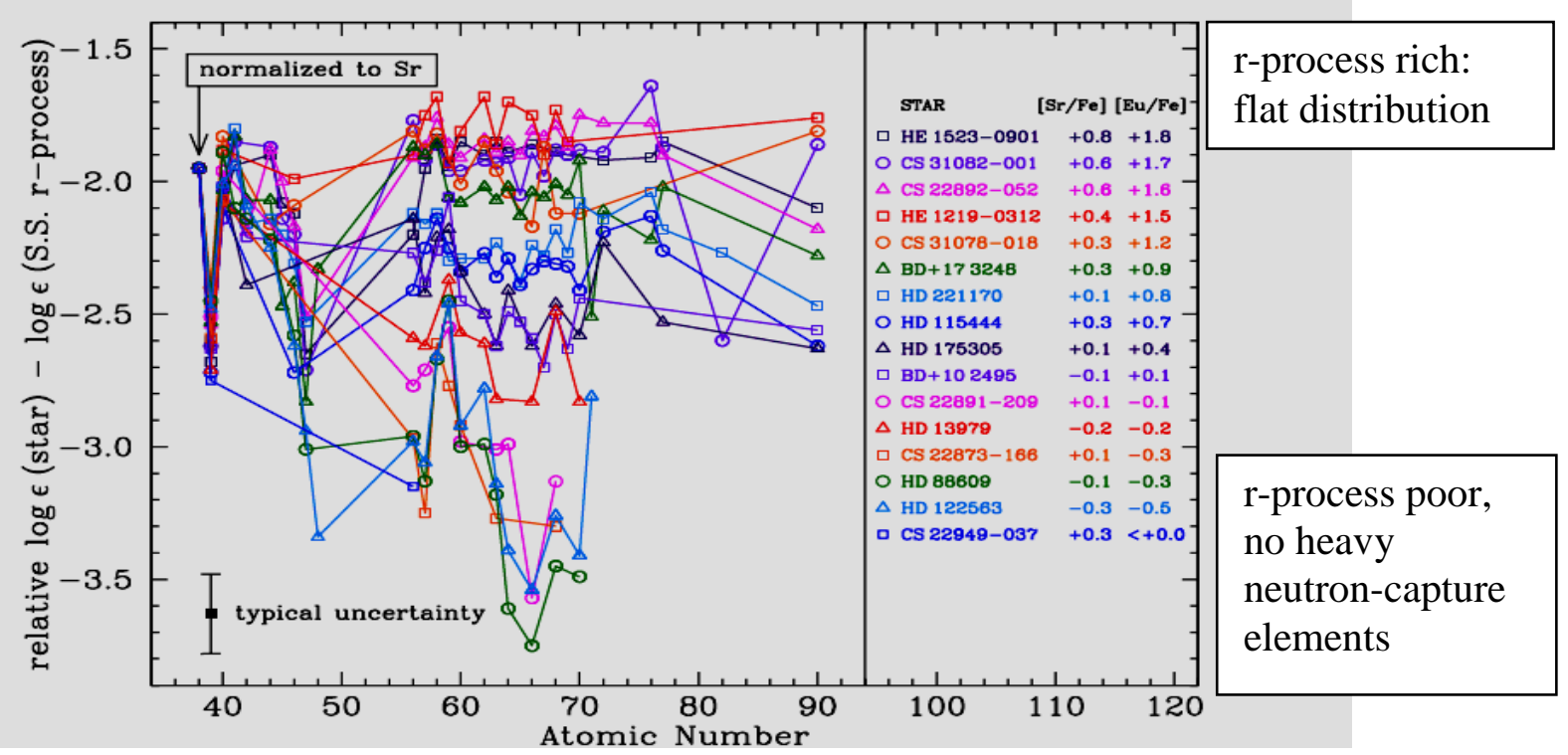

Fig. 2: This Figure shows abundances obtained for 16 r-process rich and r-process stars. It is clear that no heavy neutron-capture elements are observed in the r-process poor stars like HD122563 ( second last in the list) having [Eu/Fe]=-0.5 investigated by [7] . This Figure is adapted from [9]

The abundance distribution in the star HD122563 and similar ones suggest an incomplete rprocess, or let us say an n-capture process with a neutron density between $10^{20}-10^{24} \mathrm{~cm}^{-3}$, since the synthesis of the heavy n-capture elements needs $10^{24}-10^{28} \mathrm{~cm}^{-3}$ according to the calculations by [8] as shown in Fig.3. 

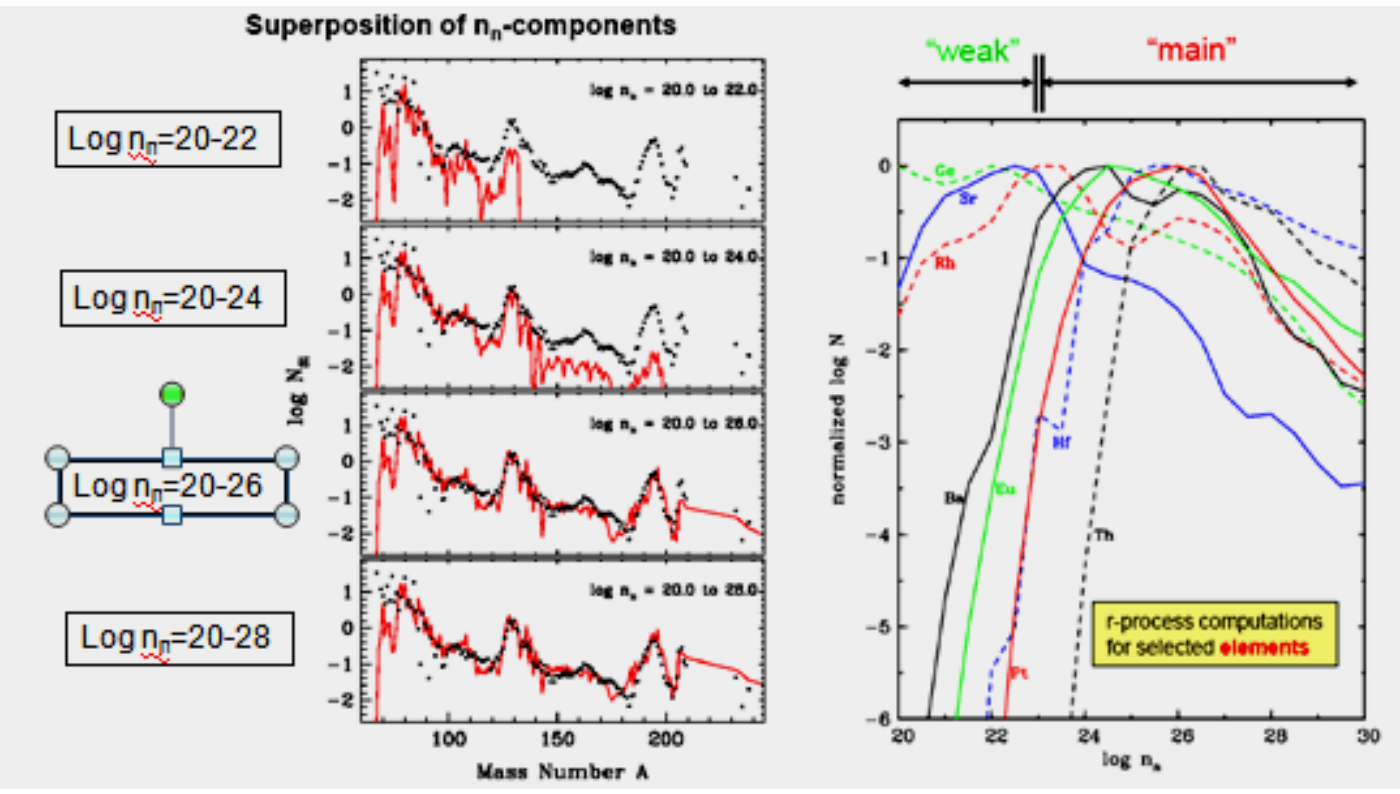

Fig.3: Distribution of the r-process components as a function of neutron density $\left(\mathrm{n}_{\mathrm{n}}\right)$ according to [8]. It is seen that the so called "weak component " of the r-process is obtained only for a neutron density in the range $\log \mathrm{n}_{\mathrm{n}}=20-22$.

In summary, the main results of the observations of neutron capture elements in the atmospheres of metal-poor stars are as follows:

- The comparison between r-process rich $([\mathrm{Eu} / \mathrm{Fe}]>1.0)$ and r-process poor

$([\mathrm{Eu} / \mathrm{Fe}]<1$ indicates that the abundances of the heavy elements ( $\mathrm{Ba}$ and above, $\mathrm{Z}=56$ ) are consistent with solar system r-process distribution. This seems to be the main r- process.

- The distribution of the lighter $(Z<56) n$-capture elements is not conform with the solar pattern. New detection of $\mathrm{Pd}, \mathrm{Ag}, \mathrm{Cd}(\mathrm{Z}=46,47,48)$ suggest a weak r-process not yet identified: is it connected to LEPP, Neutrino-proton process in core collapse Supernovae, High Entropy Wind in core collapse Supernovae, or peculiar mixing in late phases of extremely-metal poor massive stars?

\section{A Cosmic connection}

Fig. 4 contains plenty of information suggesting existence of a first generation (or pop III) stars. The infrared image (see middle panel of Fig. 4) from the Spitzer Space Telescope, reveals a distant infrared background of starlight which is expected to originate from a first generation of stars. Further evidence follows from the tremendous energy output of these very massive stars emitting highenergy photons able to re-ionize the universe. Re-ionization reminds that the universe had been previously ionized prior to recombination at about 380,000 years after the Big Bang. Data from WMAP (Wilkinson Microwave Anisotropic Probe) space craft indicate that re-ionization took place around 400 million years 
after the Big Bang suggesting that pop III stars formed at this time. An evolutionary scenario of such stars is outlined in sect. 4 .

If pop III stars were indeed very massive, their lifetime would be very short and would not survive to present day. But their explosion (see sect.4) would eject elements heavier than beryllium into space for the first time in the history of the universe. The new material would be incorporated into the new generation of stars. The presence of heavy elements in the clouds would lower the pressure significantly leading to the formation of less massive stars.

The era from recombination at 380,000 years to the first stars at 400 million years is called dark age. The only photons present at that time were those that make the present cosmic microwave background radiation. The dark age ended when the universe was filled for the first time with light from stars.

Galaxies formed more recently and can be seen at visible wavelength (right panel of Fig. 4). The dark age indicated in the Figure is an era from recombination (at $380,00 \mathrm{yr})$ to the first stars at 400 million yrs.

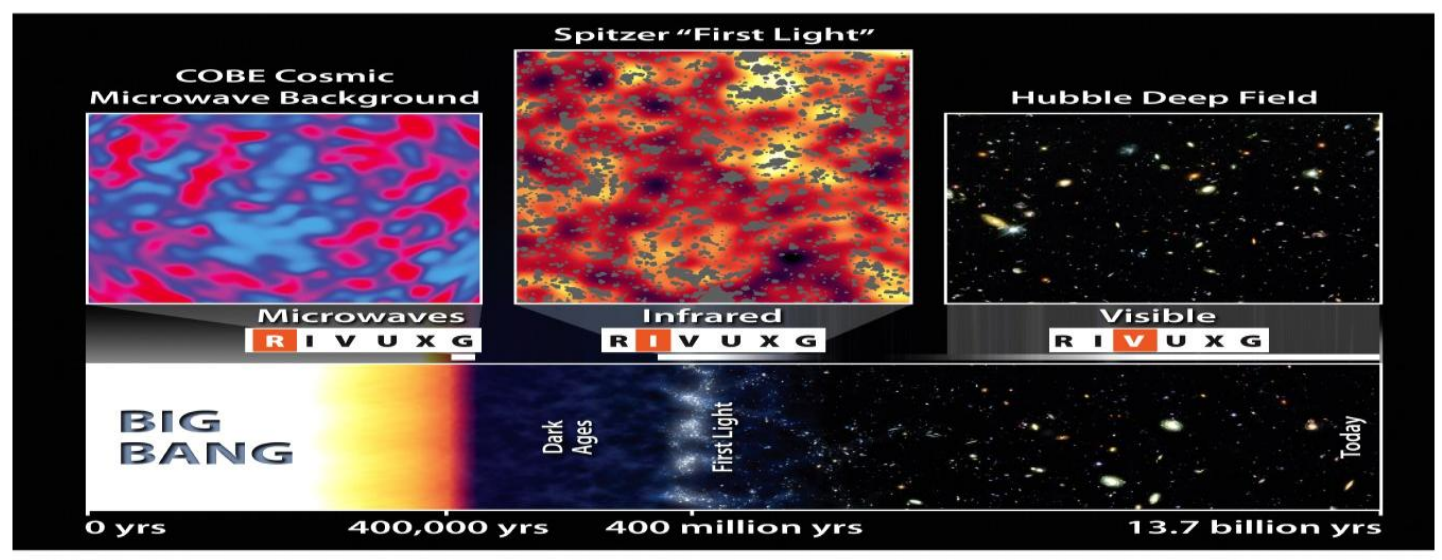

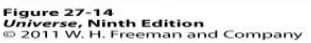

Figure 4: Timeline of the light in the universe. The oldest light that we can see today is the cosmic background radiation, which comes from the time 380,000 years after the Big Bang when the universe became transparent. This light has a redshift $\mathrm{z}=1100$ and appears as microwave. Some 400 million years later at $\mathrm{z}=11$, the first stars appeared: their light is now shifted to infrared wavelengths. Galaxies formed more recently and are seen at visible wavelength. This Figure is adapted from $[10]$.

\section{Evolutionary Scenarios of massive and very massive stars}

A detailed discussion of the evolution of massive or very massive stars is beyond the scope of the present contribution. We just present a short discussion dealing with the explosion of very massive stars leading to the called "Pair Creation Supernovae", and describe a scenario of late evolution of massive stars of extremely low metallicity and try to connect this scenarios to the formation of the light neutron-capture elements. 


\subsection{Pair Creation Supernova (PCSN)}

The evolution of stars of different masses may be summarized as follows. Stars of masses up to about $8 \mathrm{M}_{\odot}$ evolve through the red giant phase, asymptotic giant branch phase, planetary nebula phase to become white dwarfs. Stars of masses $8<\mathrm{M} / \mathrm{M}_{\odot}<140$ evolve to become super giants and suffer iron-core collapse and may explode as type II supernovae leaving a neutron star or a black hole behind. The limit on the initial mass for forming a neutron star is about $30 \mathrm{M}_{\odot}$. Stars in the mass range $140<\mathrm{M} / \mathrm{M}_{\odot}<260$ develop very massive carbon-oxygen cores of $64<\mathrm{M} / \mathrm{M}_{\odot}<133$ which evolve through the region of electron-positron pair creation. Finally, stars above $260 \mathrm{M}_{\odot}$ end up forming black holes. This discussion can be found in [11] and an up-dated overview is given by [12].
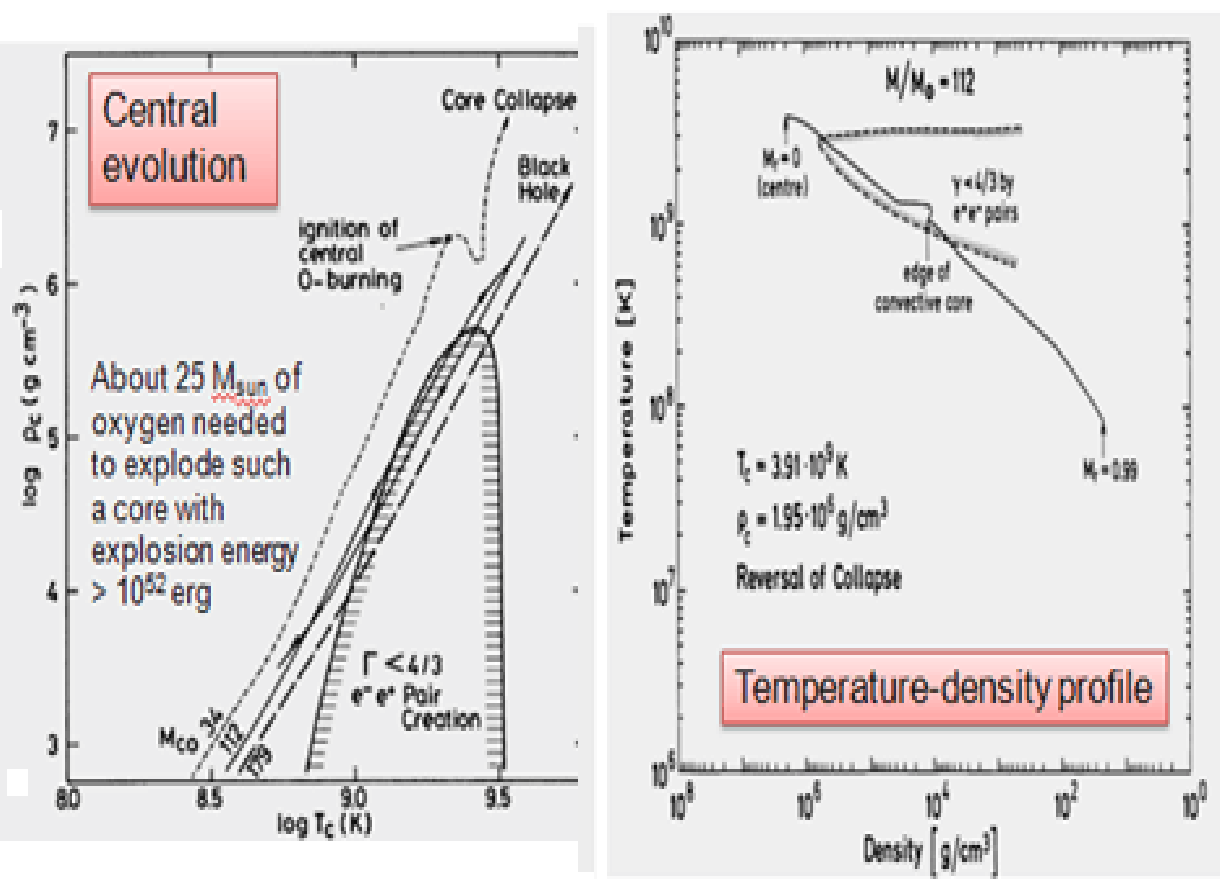

Fig. 5: left panel shows the evolution of carbon-oxygen cores of masses 34,112 and $179 \mathrm{M}_{\odot}$. The dashed curve encloses the domain of pair creation instability, where the adiabatic index $\Gamma<4 / 3$ (see text). Right panel shows that a significant part the $112 \mathrm{M}_{\odot}$ core lies in the instability domain.

Concentrating on the pair creation instability, we describe the basic mechanism and refer to more details in [11], [12], [13] and references therein. Fig. 5 (left panel), taken from the paper by [11], shows the evolution of several $\mathrm{C} / \mathrm{O}$ cores in the central density $\left(\rho_{c}\right)$ and central temperature $\left(\mathrm{T}_{\mathrm{c}}\right)$ plane. In particular, the $\mathrm{C} / \mathrm{O}$ core of $112 \mathrm{M}_{\odot}$ which originates from an initial mass of $200 \mathrm{M}_{\odot}$ (see [11]) evolves into the domain of pair 
creation instability, where a significant part of the core lies in that domain (see right panel of Fig. 5). The ensuing collapse leads to explosive oxygen burning consuming an amount of $25 \mathrm{M}_{\odot}$ enough to blow up the whole core in a brilliant supernova releasing $10^{52}$ ergs according to the calculation by [11].

More details about this scenario may be useful. As seen in Fig. 5, the evolution of the massive $\mathrm{C} / \mathrm{O}$ cores proceeds toward higher temperatures and relatively low densities, such that electron positron pairs are created in equilibrium by the intense radiation field according to reaction $2 \gamma \leftrightarrow e^{-}+e^{+}$

Although the mean energy of photons is about kT, there are enough photons in the tail of the Planck's distribution that can create these pairs even at $10^{9} \mathrm{~K}$. The creation of new particles decreases the radiation pressure and these new particle do not add immediately their contribution to the total pressure. At high densities $\Gamma$ exceeds $4 / 3$ because the electrons become degenerate, while at high temperature electrons and positrons become relativistic so that the energy gap for the pair creation becomes unimportant. The net effect is a bounded region in which the adiabatic index $\Gamma$ drops below 4/3. as displayed in Fig.5.

So far we have argued that that the PCSNe may be associated with pop III stars. However, new insight concerning these events came up recently indicating that these events may occur in the local universe. It is surprising that there are candidates of such supernovae, namely SN2007bi discovered by [14] and SN2006gy found by [15]. The light curve of SN2007bi is displayed in Fig. 6. According to the analysis of the light curve by [14], more than $3 \mathrm{M}_{\odot}$ of ${ }^{56} \mathrm{Ni}$ are needed to power the late behaviour of the light curve. It is argued that the slow rise time of about 70 days of the light curve and the expansion velocity of $12000 \mathrm{~km} / \mathrm{s}$ suggest the explosion of a very massive object of about $100 \mathrm{M}_{\odot}$ and explosion energy in excess of $10^{52} \mathrm{erg}$. We also mention that the SN2007bi seems to have occurred in a medium of sub solar metallicity of about $1 / 3$ of the solar value (see [16] for details and references).

There are several implications of the discovery of SN2007bi. (i) The estimated high core mass is in conflict with the commonly used mass loss rates as a function of metallicity, (ii) Regardless the correct description of mass loss, the data indicate that an extremely massive stars $\left(>150 \mathrm{M}_{\text {sun }}\right)$ are formed in the local universe in a dwarf galaxy with a metallicity $12+\log [\mathrm{O} / \mathrm{H}]=8.25$ (less than $1 / 10$ of the Sun's metallicity, (iii) Future missions like the NASA's James Webb Space telescope will help to estimate the contribution of these events to the chemical evolution in the early universe. 


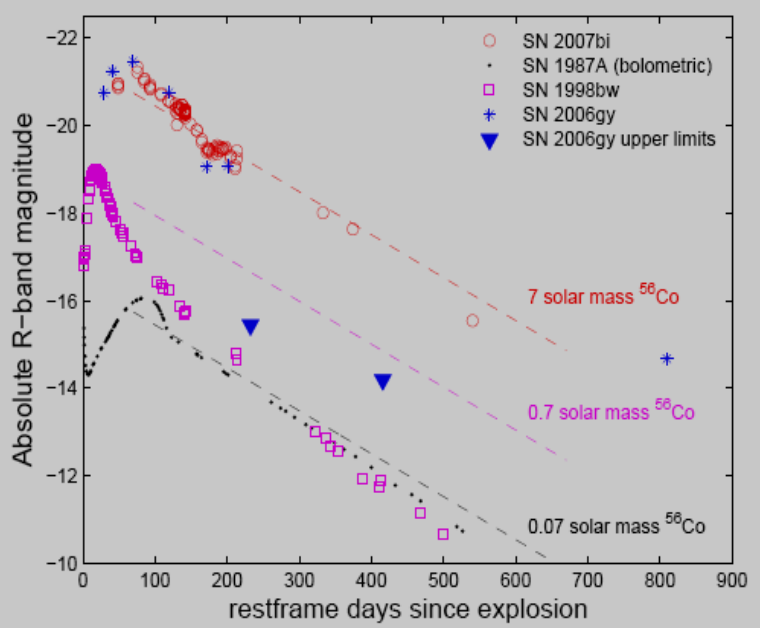

Fig. 6: R-band light curve of the super luminous supernova SN2007bi with a peak magnitude of -21.3 according to the observations by [14].

\subsection{Nucleosynthesis in Pair Creation Supernovae}

Updated nucleosynthesis calculations have been done by [12]. The main results of these calculations indicate that no elements heavier than zinc $(Z=30)$ are formed, and no r-process, or s-process products. The main products are due to explosive oxygen burning, namely $\mathrm{Si}, \mathrm{S}, \mathrm{Ar}, \mathrm{Ca}$ in almost solar distribution. Elements of odd nuclear charges are deficient, because the massive $\mathrm{C} / \mathrm{O}$ cores evolve almost directly to oxygen burning without creating a neutron excess. In other words, only alpha elements are mainly produced in these explosions. One may conclude that the PCSNe are not responsible for the formation of the light neutron-capture elements we have described above. In other words, they are not candidates for the LEPP we mentioned above.

\subsection{Peculiar Neutron-capture Scenario}

As a final part of this contribution we discuss a possibility of producing some of the light neutron-capture elements without claiming that such scenario is associated with the LEPP. We may consider the evolution of massive stars of $25 \mathrm{M}_{\odot}$ to explain the peculiarity of the evolution of massive stars at extremely low metallicity. 


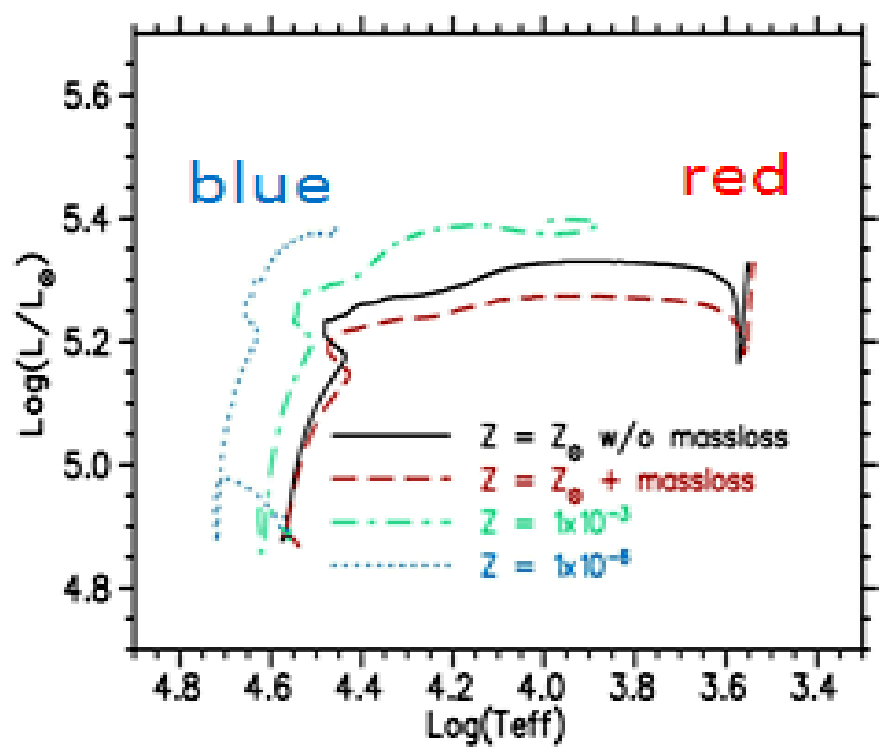

Fig. 7: Theoretical Herztsprung-Russell diagram for a $25 \mathrm{M}_{\odot}$ star of variable initial chemical composition marked by the value of metallicity $Z$ ( see [17] for details). The dashed-dotted curve represents the evolutionary track for $Z=10^{-3}$, while dotted curve corresponds to $Z=10^{-6}$.

The Hertzsprung-Russell diagram (referring to as HR diagram) shows the following characteristics:

- The zero-age main sequence is shifted to higher effective temperatures as the metallicity $\mathrm{Z}$ decreases. This is a direct consequence of the reduced metallicity, since the star is forced to contract and heats up so that the hydrogen-burning by the CNO cycle operates at higher temperature to establish hydrostatic and thermal equilibrium.

- The luminosity of the star on the main sequence becomes higher when $\mathrm{Z}$ decreases. This is mainly due to the reduced opacity.

- An important point for our discussion is that the tracks of the metal-poor stars (with lower Z) have the tendency to remain confined to the blue part of the HR diagram and this occurs for $Z<10^{-3}$ as seen in Fig.7. How is this behavior related to the internal structure? This is shown in the following with the help of Fig. 8 and 9.

In Fig. 8 we compare the change of internal structure as a function of time for the $25 \mathrm{M}_{\odot}$ star of initial solar-like metallicity, $\mathrm{Z}=0.02$, with that of $\mathrm{Z}=10^{-3}$ The details of this evolution is described elsewhere (see [17]) and references therein). Here, we emphasize the point relevant to our present discussion, namely that the hydrogen burning shell remain convective all the way in case of $Z=10^{-3}$, while the hydrogen-burning shell in the solar-like case, ceases to be convective already during core helium burning. Note that the metal-poor star does not develop a convective envelope, because it does not evolve to the red giant phase (see Fig. 7), where this convective envelope is formed. In other words, the hydrogen burning shell is not able to inflate the more compact metal-poor star. 

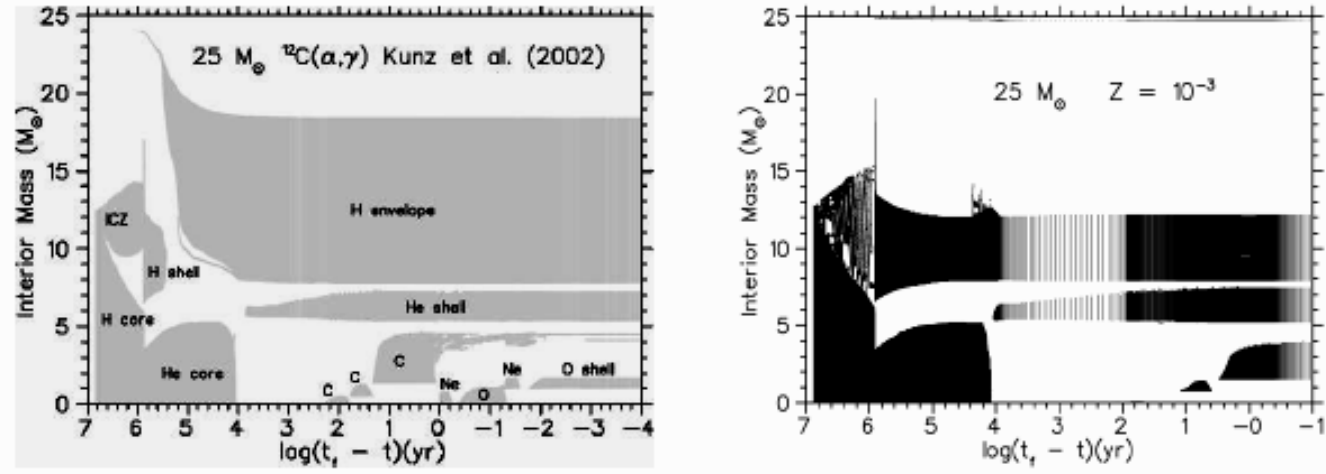

Fig.8: Change of internal structure as a function of time of a $25 \mathrm{M}_{\odot}$ star of initial solar-like metallicity (Z) (left panel) and of initial $Z=10^{-3}$ (1/20 solar). Note that the time axis denotes the time of the last calculated stellar model minus that of the initial one on the main sequence

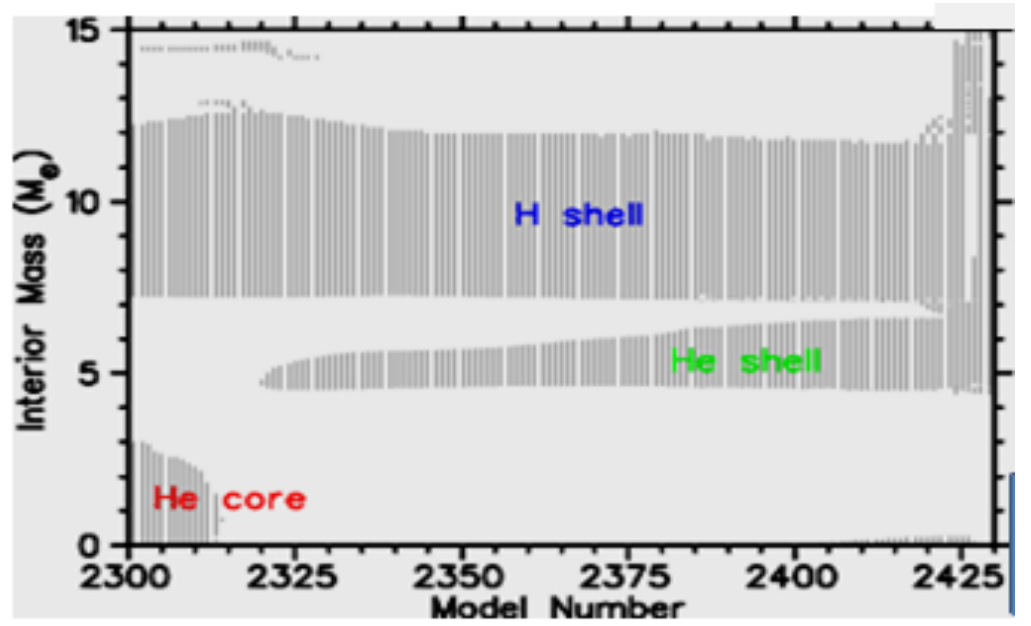

Fig.9: Same as Fig.8 but for an initial metallicity of about $10^{-5}$. The evolution of this star is shown up to the merging of the hydrogen-burning shell with the helium-burning shell. Such calculation is still in preparation by [17].

Continuing to describe this issue, we see in Fig. 7 that the track with initial $Z=10^{-6}$ remains just in the blue part of the HR diagram. The evolution of the internal structure with time of the same $25 \mathrm{M}_{\odot}$ star is depicted in Fig. 9. This case is a calculation for the $25 \mathrm{M}_{\odot}$ star with initial metallicity close to $\mathrm{Z}=10^{-5}$. An interesting feature occurs after the core helium burning when the star has a convective hydrogen-burning shell and helium-burning shell. According to this preliminary calculations (see [17]), the hydrogen-burning shell expands inward and merge with the helium-burning shell beneath it. This would mean mixing protons into a carbon-rich region, where the carbon has been synthesized by shell-helium burning. The mixed protons can initiate the reaction ${ }^{13} \mathrm{C}(\alpha, n){ }^{16} \mathrm{O}$, so that a primary neutron source would be available for the production of heavy elements. A preliminary investigation shows that neutron-capture heavy elements with $Z=38$ to 50 can be produced. In particular, a primary production of 
$\mathrm{Sr}$ is possible in this context. But, further work is needed to establish this attractive scenario which can be linked to the LEPP we have mentioned above.

\section{Concluding Remarks}

Evolution of early stars linked to nucleosynthesis of heavy elements turns out to be a link to Near-field cosmology (understanding galaxy formation). It is a challenging topic and a revival of the importance of stellar evolution as a fundamental cornerstone of modern Astrophysics. The evolution of the neutron-capture elements traces back the chemical evolution of the galaxy and bring us back to the dark ages to become more enlightened and overcome our ignorance. More work is needed to understanding the nature of the formation of early heavy elements.

Acknoledgement: I thank the organiser of the $6^{\text {th }}$ European Summer School on Experimental Nuclear Astrophysics for their support, and the URB at the American University of Beirut (AUB) for supporting the trip to Santa Tela.

\section{References}

[1] V. Bromm, et al., Nature, 2009, 459, 29

[2] C. Sneden et al., ApJ, (2003), 591, 936

[3] Hill etal. (2002), A\&A, 387, 560

[4] Crawford et al , (1998). ApJ, 116, 2489

[5] Travaglio et al , (2004), ApJ, 601, 864

[6] Montes et al, (2007), ApJ, 671, 1685

[7] Honda et al , (2006), ApJ, 643, 1180

[8] Kratz et al, (2007), ApJ, 662, 39

[9] J.J. Cowan et al, (2011), xiv:1106.11091/1

]10] R.A. Freedman, R.M. Geller, W.J. Kaufmann III, 2011 in Universe, $9^{\text {th }}$ edition, (Freeman: New York), p. 735

[11] W. Ober, M.F. El Eid, K. Fricke, 1983, A\&A, 119, 61

[12] A. Heger, S.E. Woosley, 2002, ApJ, 567, 532

[13] N. Langer, 20098, Nature, 462, 579

[14] Gal-Yam et al, Nature, 2009, 462, 624

[15] Smith et al, ApJ, 2007, 666, 1116

[16] Langer, N. Nature , 2009, 462, 579

[17] M. El Eid, L.-S The , B.S. Meyer , 2009, Space Sci. Rev. , 147, 1

[18] L.-S. The, M.F El Eid, B.S Meyer, in preparation 